\title{
Neural basis of episodic memory in the intermediate term after medial temporal lobe resection
}

\author{
Woorim Jeong, PhD, ${ }^{1,2}$ Hyeongrae Lee, PhD, ${ }^{3}$ June Sic Kim, PhD, ${ }^{4}$ and \\ Chun Kee Chung, MD, PhD $1,2,4$
}

\begin{abstract}
${ }^{1}$ Neuroscience Research Institute, Seoul National University College of Medicine; ${ }^{2}$ Department of Neurosurgery, Seoul National University Hospital; ${ }^{3}$ Department of Mental Health Research, National Center for Mental Health; and ${ }^{4}$ Department of Brain and Cognitive Sciences, Seoul National University College of Natural Sciences, Seoul, Korea
\end{abstract}

\begin{abstract}
OBJECTIVE How the brain supports intermediate-term preservation of memory in patients who have undergone unilateral medial temporal lobe resection (MTLR) has not yet been demonstrated. To understand the neural basis of episodic memory in the intermediate term after surgery for temporal lobe epilepsy (TLE), the authors investigated the relationship between the activation of the hippocampus (HIP) during successful memory encoding and individual memory capacity in patients who had undergone MTLR. They also compared hippocampal activation with other parameters, including structural volumes of the HIP, duration of illness, and age at seizure onset.

METHODS Thirty-five adult patients who had undergone unilateral MTLR at least 1 year before recruiting and who had a favorable seizure outcome were enrolled (17 left MTLR, 18 right MTLR; mean follow-up $6.31 \pm 2.72$ years). All patients underwent a standardized neuropsychological examination of memory function and functional MRI scanning with a memory-encoding paradigm of words and figures. Activations of the HIP during successful memory encoding were calculated and compared with standard neuropsychological memory scores, hippocampal volumes, and other clinical variables.
\end{abstract}

RESULTS Greater activation in the HIP contralateral to the side of the resection was related to higher postoperative memory scores and greater postoperative memory improvement than the preoperative baseline in both patient groups. Specifically, postoperative verbal memory performance was positively correlated with contralateral right hippocampal activation during word encoding in the left-sided surgery group. In contrast, postoperative visual memory performance was positively correlated with contralateral left hippocampal activation during figure encoding in the right-sided surgery group. Activation of the ipsilateral remnant HIP was not correlated with any memory scores or volumes of the HIP; however, it had a negative correlation with the seizure-onset age and positive correlation with the duration of illness in both patient groups.

CONCLUSIONS For the first time, a neural basis that supports effective intermediate-term episodic memory after unilateral MTLR has been characterized. The results provide evidence that engagement of the HIP contralateral rather than ipsilateral to the side of resection is responsible for effective memory function in the intermediate term (> 1 year) after surgery in patients who have undergone left MTLR and right MTLR. Engagement of the material-specific contralesional HIP, verbal memory in the left-sided surgery group, and visual memory in the right-sided surgery group were observed. https://thejns.org/doi/abs/10.3171/2018.5.JNS18199

KEYWORDS medial temporal lobe resection; hippocampus; temporal lobe epilepsy; episodic memory; intermediateterm memory outcome; functional MRI

$\Lambda$ LTHOUGH postoperative episodic memory decline is a major complication of medial temporal lobe resection (MTLR), which is the standard treatment for medically intractable medial temporal lobe epilepsy (TLE), ${ }^{4,12}$ two-thirds of patients with TLE maintain stable or even improved episodic memory function within the first 1-2 years after surgery., ${ }^{3,21}$ With the longstanding history of neuropsychological evaluation in epilepsy surgery programs, increasing studies are now investigating the intermediate- to long-term outcomes of memory function. ${ }^{28}$

ABBREVIATIONS AFNI = Analysis of Functional Neurolmages; EPI = echo-planar imaging; fMRI = functional MRI; FSIQ = full-scale IQ; HIP = hippocampus; KAVLT = Korean version of the Rey Auditory Verbal Learning Test; KCFT = Korean version of the Rey Complex Figure Test; KWAIS = Korean Wechsler Adult Intelligence Scale; LMTLR = left MTLR; MNI = Montreal Neurological Institute; $M Q=$ memory quotient; $M T L=$ medial temporal lobe; $M T L R=M T L$ resection; PIQ = performance IQ; RMTLR = right MTLR; $R O I$ = region of interest; TLE = temporal lobe epilepsy; $T R=$ repetition time; VIQ = verbal IQ.

SUBMITTED January 23, 2018. ACCEPTED May 2, 2018.

INCLUDE WHEN CITING Published online October 26, 2018; DOI: 10.3171/2018.5.JNS18199. 
These studies have reported that there is no further decline and even improvement in episodic memory function at $1-10$ years after MTLR., ${ }^{1,2,19,28}$

For the purpose of better understanding memory function in TLE patients, extensive studies have investigated the neural basis of episodic memory by using functional MRI (fMRI) techniques. ${ }^{4,11}$ Most previous studies, however, were conducted only to understand the neural basis of preoperative memory function and/or to unveil the preoperative predictive factors for postoperative memory outcomes by using preoperative data. A postoperative neural basis associated with memory function is relatively less understood, but understanding this matter could help with developing not only better prediction models of memory outcome after MTLR but also a deeper understanding of human memory systems in general.

Moreover, although the stability of intermediate-term memory outcomes has been well studied, the manner in which the brain supports normal episodic memory function in the intermediate term ( $>1$ year) after MTLR has not been investigated. Only a few studies have investigated the neural basis of episodic memory function within 1 year of TLE surgery. 5,,16,22 Two previous postoperative studies, unfortunately, did not compare memory scores with solely postoperative brain activation. ${ }^{5,16}$ Two other studies directly correlated postoperative neural responses with memory performance and emphasized the role of the hippocampus (HIP) on the side contralateral to the site of resection for maintaining postoperative episodic memory function. ${ }^{7,22}$ However, one previous study reported that hippocampal activation displayed dynamic changes from 3 to 12 months after surgery, ${ }^{22}$ which makes it difficult to conclude how similar the neural basis of intermediateterm memory outcomes are to the neural basis during the time up to 12 months after surgery. In addition, although other clinical parameters, such as the duration of illness and age at seizure onset, were shown to influence memory-related preoperative brain activations ${ }^{23}$ no study has yet investigated the effect of these factors on postoperative brain function.

Therefore, the purpose of the present study was to characterize the neural basis that supports effective intermediate-term episodic memory function ( $>1$ year) after a unilateral MTLR. To provide intermediate-term follow-up neuroimaging evidence of memory outcomes, the fMRI activation patterns in regions of interest (ROIs) of a bilateral HIP during memory were compared with the neuropsychological memory performance data, as well as structural volumes of the HIP and other clinical variables, including duration of illness and age at seizure onset, in patients who underwent unilateral MTLR at least 1 year before fMRI memory scanning.

\section{Methods \\ Patients}

Patients who underwent unilateral MTLR for medically intractable TLE at Seoul National University Hospital were retrospectively recruited. Inclusion criteria were individuals who: 1) underwent unilateral MTLR at least 1 year before recruitment, 2) had an IQ over 70 on the most recent Korean Wechsler Adult Intelligence Scale
(KWAIS) test, and 3) were between 19 and 50 years of age at the time of recruiting. A total of 43 patients agreed to participate in this study. Two patients with an unfavorable seizure outcome (Engel class III $)^{10}$ and 3 patients who had severe dental metallic artifacts were excluded. To ensure proper understanding and engagement of fMRI memory tasks, 3 patients who exhibited low memory capacity on a standard neuropsychological test ( 2 borderline, 1 impairment) were also excluded. Overall, 35 patients were included in this study (17 left and 18 right TLE) (Table 1). There were no statistically significant differences between the patient groups in terms of age $(p=0.481)$, education year $(p=0.976)$, age at seizure onset $(p=0.948)$, age at the time of surgery $(\mathrm{p}=0.418)$, duration of illness $(\mathrm{p}=0.622)$, and follow-up duration $(\mathrm{p}=0.782)$.

All patients underwent either a selective amygdalohippocampectomy or anterior temporal wedge resection with amygdalohippocampectomy: 6 of 17 in the left MTLR (LMTLR) group and 3 of 18 in the right MTLR (RMTLR) group underwent selective amygdalohippocampectomy. A previous report described the surgical procedures. ${ }^{8}$ Groups were not separated by surgical procedures since it is still inconclusive as to whether memory outcome differs according to different surgery types or the extent of resection. ${ }^{20}$ Moreover, memory outcome was not different according to the surgery type (Mann-Whitney U-test) in this study. This study was approved by the institutional review board of Seoul National University Hospital, and all patients provided informed consent.

\section{Neuropsychological Testing}

The standardized neuropsychological testing instrument KWAIS (full-scale IQ [FSIQ]; verbal IQ [VIQ]; and performance IQ [PIQ] ${ }^{29}$ and the Korean version of the Rey-Kim memory test (the Korean version of the Rey Auditory Verbal Learning Test [KAVLT] and the Korean version of the Rey Complex Figure Test ${ }^{15}$ [KCFT]) were administered before and after surgery. Postoperative neuropsychological tests were performed on a separate day within 1 month from the day of the fMRI. Age-adjusted memory quotient (MQ) subtest scores of the KAVLT immediate recall and KAVLT delayed recall for verbal memory and KCFT immediate recall and KCFT delayed recall for visual memory were used. To estimate the postoperative changes in cognitive ability, the preoperative scores were subtracted from the postoperative scores. All statistical analyses of the behavioral data in the present study were calculated using SPSS 19.0 software (IBM Corp.); significance was set at $\mathrm{p}=0.05$. A Student t-test, pairedsample t-test, and Pearson correlation analysis were used as appropriate.

\section{MRI Data Acquisition}

All patients were examined before surgery using either a GE 1.5- or 3-T MRI unit (GE Horizon Echospeed) or a Siemens 1.5-T scanner (Siemens Avento system). T1weighted or 3D gradient echo coronal T1-weighted images were used for preoperative hippocampal volume calculation. Postoperative MR images were acquired on a research-dedicated 3-T MAGNETOM Trio Tim Syngo 
(Siemens) using a 32-channel head coil. A series of highresolution anatomical T1-weighted images were obtained with a 3D turbo-FLASH sequence (TR $1670 \mathrm{msec}$, TE $1.89 \mathrm{msec}$, field of view $250 \times 250 \mathrm{~mm}$, flip angle $9^{\circ}$, voxel size $1.0 \times 1.0 \times 1.0 \mathrm{~mm}^{3}$ ) before the functional scans. Functional data were acquired using a T2*-weighted gradient echo planar imaging sequence ( 36 axial slices, slice thickness $3.4 \mathrm{~mm}$ [no gap], TR $2750 \mathrm{msec}$, TE $30 \mathrm{msec}$, field of view $220 \times 220 \mathrm{~mm}$, flip angel $80^{\circ}$, voxel size 3.4 $\times 3.4 \times 3.4 \mathrm{~mm}^{3}$, interleaved).

\section{Memory Task Paradigm}

All patients performed a memory-encoding task during fMRI scanning. Verbal stimuli (single concrete nouns) and visual stimuli (black-and-white unnameable abstract figures) were presented on an MRI-compatible screen viewed through a mirror. All stimuli were presented using the E-prime software (version 2.0, Psychology Software Tools Inc.). Cross-hair fixation was presented for $15 \mathrm{sec}-$ onds after every presentation of 20 items (10 words and 10 figures, 1 item for 3 seconds). A total of 100 words and 100 figures were presented in two separate scanning runs. About 30 minutes after encoding, all patients performed out-scanner recognition tasks. The same 100 items intermixed with an additional 50 novel words/figures were shown in random order. Patients had to select whether the presented item was old, familiar, or novel. Recognition accuracy (\%) was calculated for both words and figures as the hit rate (stimuli correctly remembered) - the false alarm rate (novel stimuli incorrectly tagged as remembered).

\section{Functional Imaging Data Analysis}

Functional imaging data were analyzed using Analysis of Functional NeuroImages (AFNI) software (https://afni. nimh.nih.gov/afni/, version: AFNI_16.0.00). The first two repetition times (TRs) of the echo-planar imaging (EPI) time series were discarded for magnetization stabilization. Motion correction by rigid body registration of the EPI images to the first truncated EPI volume and slice timing correction were performed. Then, the anatomical T1weighted images were aligned to the mean EPI volume via an affine transformation. In order to reduce the error imposed by the surgical cavity, the local Pearson correlation cost function, which had been shown to be superior, for this purpose, to more general multimodal cost functions, ${ }^{18,30}$ was used. Spatial normalization was then performed to affine transform data into the Montreal Neurological Institute (MNI) stereotactic space using the MNI avg152T1 template. All voxels were resampled as a $2 \times 2$ $\times 2-\mathrm{mm}$ size by linear interpolation. Mean-based intensity normalization was performed after spatial smoothing using a Gaussian filter with 6-mm full width at half maximum.

After preprocessing, the hemodynamic response starting from the stimulus onset to 13.75 seconds was estimated using a cubic spline basis function separately for words remembered, words familiar, words forgotten, figures remembered, figures familiar, and figures forgotten. To correct motion-related artifacts, 6 motion parameters
TABLE 1. Summary of patient demographics

\begin{tabular}{|c|c|c|}
\hline Variable & $\operatorname{LMTLR}(\mathrm{n}=17)$ & $\operatorname{RMTLR}(\mathrm{n}=18)$ \\
\hline Mean age, yrs & $34.53(6.49)$ & $32.83(7.52)$ \\
\hline $\operatorname{Sex}(M / F)$ & $8: 9$ & $10: 8$ \\
\hline Mean education, yrs & $14.35(1.93)$ & $14.33(1.81)$ \\
\hline Mean seizure onset, yrs & $14.71(9.98)$ & $14.94(10.52)$ \\
\hline Mean duration of illness, yrs & $13.24(9.40)$ & $11.56(9.07)$ \\
\hline Mean age at surgery, yrs & $27.94(6.11)$ & $26.50(6.68)$ \\
\hline \multicolumn{3}{|l|}{ Follow-up, yrs } \\
\hline $1-2$ & 1 & 0 \\
\hline $2-5$ & 3 & 7 \\
\hline $5-10$ & 11 & 10 \\
\hline$>10$ & 2 & 1 \\
\hline Mean & $6.44(2.74)$ & $6.18(2.78)$ \\
\hline \multicolumn{3}{|l|}{ Surgical outcome (\%) } \\
\hline Engel class I & $15(88)$ & $16(89)$ \\
\hline Engel class II & $2(12)$ & $2(11)$ \\
\hline \multicolumn{3}{|l|}{ No. of antiepileptic drugs (\%) } \\
\hline 0 & $8(47)$ & $12(67)$ \\
\hline 1 & $7(41)$ & $5(28)$ \\
\hline 2 & $2(12)$ & $1(5)$ \\
\hline \multicolumn{3}{|l|}{ Pathology } \\
\hline Hippocampal sclerosis & 8 & 5 \\
\hline $\begin{array}{l}\text { Focal cortical dysplasia } \\
\text { type I or II }\end{array}$ & 1 & 2 \\
\hline Dual pathology & 7 & 9 \\
\hline Tumor & 1 & 2 \\
\hline
\end{tabular}

Values are presented as the number of patients or as the mean (SD).

were included in the first-level general linear models and censored TRs from the general linear models based on threshold of framewise displacement $>0.9$. Framewise displacement is the sum of the absolute value of temporal differences across the 6 motion parameters. The mean percentage of signal changes was calculated by averaging the beta from the second to fifth points (2.75-11 seconds) of the estimated response for each patient.

For defining the HIP as ROI individually, bilateral HIPs were segmented in each patient's high-resolution structural space by using automatic and manual segmentation methods (Fig. 1). Both automatic and manual segmentation were chosen because automatic segmentation could not accurately detect the posterior parts of the HIP in some patients. Automatic segmentation was performed using the STEPS (Similarity and Truth Estimation for Propagated Segmentations) algorithm, ${ }^{6}$ available online at http://cmictig.cs.ucl.ac.uk/niftyweb/. This method has been proven to delineate the HIP accurately in TLE patients with hippocampal lesion. ${ }^{27}$ The areas that were not detected in automatic segmentation were manually delineated using AFNI software by one of the authors (W.J.) and confirmed by a neurosurgeon (C.K.C.) who is an expert in HIP anatomy. The determination of the anatomical boundaries of hippocampal formation was based on previously developed protocols..$^{13}$ In the present study, we 
defined resection volume as hippocampal volume differences between the time of study participation (postoperatively) and preoperatively.

\section{Extracting Hippocampal Activation}

Hippocampal activation was calculated by averaging the mean percentage of signal changes of the activated voxels in the hippocampal ROIs during encoding of the subsequently remembered stimuli (correct) compared to the subsequently forgotten or familiar stimuli (incorrect). The left and right hippocampal activations were then correlated with the clinical variables using the Pearson correlation coefficient. Examples of the activated voxels in the hippocampal ROIs during the word and figure encoding (correct > incorrect) are shown in Fig. 2.

\section{Results}

\section{Neuropsychological Performance}

The preoperative IQ of one LMTLR patient (case 14) was excluded from the analyses because that patient's IQ was examined by the KWAIS-IV, which has a different scoring system than that of the KWAIS. For general intelligence, pre- or postoperative IQ scores were not significantly different between the LMTLR and RMTLR groups $(p>0.1)$. When the pre- and postoperative IQ scores were compared, there was a significant postoperative increase in both LMTLR $(\mathrm{p}<0.001)$ and RMTLR $(\mathrm{p}<0.05$ for FSIQ and VIQ, $\mathrm{p}<0.001$ for PIQ) groups (Table 2).

For memory performance, both pre- and postoperative full-scale MQs and the preoperative MQ subtests were not significantly different between the two patient groups ( $\mathrm{p}>$ $0.05)$. Postoperatively, both the verbal immediate and delayed recall scores were significantly lower in the LMTLR group than the RMTLR group $(p<0.05)$. Both the visual immediate and delayed recall scores were significantly lower in the RMTLR group than the LMTLR group ( $p$ $<0.05)$.

Similar to IQ, the postoperative full-scale MQ scores significantly increased in both the LMTLR $(\mathrm{p}<0.05)$ and RMTLR ( $<0.001)$ groups compared to the preoperative baseline scores. Both groups exhibited significant postoperative increases in all verbal and visual subtests of MQ, except for visual immediate recall in the RMTLR group (LMTLR, $p<0.05$ for all subtests; RMTLR, $p<0.001$ for verbal delayed recall and $p<0.05$ for verbal immediate and visual delayed recall).

\section{Hippocampal Volumes}

The resection volume and both pre- and postoperative volumes of the HIP ipsilateral and contralateral to the side of resection were not statistically different between LMTLR and RMTLR groups ( $\mathrm{p}>0.05$, Table 3$)$. When hippocampal volumes were correlated with neuropsychological memory scores, no statistically significant relationship was detected between preoperative volumes and preoperative memory, between resection volume and postoperative changes in memory, or between postoperative volumes and postoperative memory scores. In addition, postoperative volumes were not related to hippocampal activations $(\mathrm{p}>0.05)$.

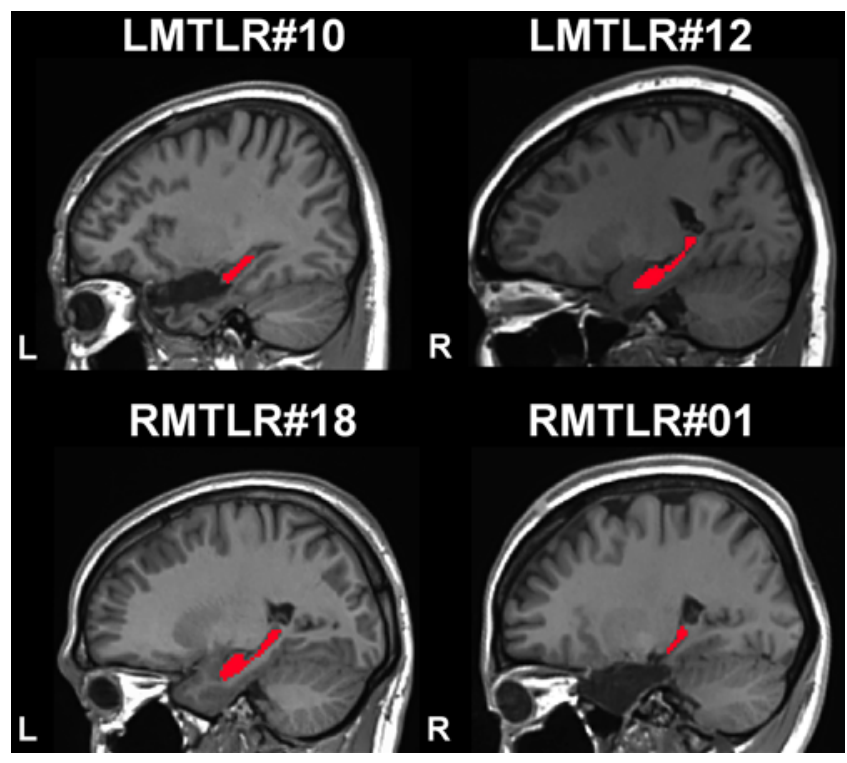

FIG. 1. Examples of manually drawn hippocampal ROls. $L=$ left; $R=$ right. Figure is available in color online only.

\section{Behavioral Results}

There were no statistically significant differences in word (mean accuracy for LMTLR, 48.75\% $\pm 22.99 \%$; mean accuracy for RMTLR, $56.00 \% \pm 17.52 \%$; $\mathrm{p}=0.306$ ) or figure recognition performance (mean accuracy for LMTLR, $18.35 \% \pm 14.19 \%$; RMTLR, $15.33 \% \pm 14.14 \% ; p=0.533$ ) between the two patient groups. The results confirm that all patients understood and performed our memory task well (chance level 0\%).

\section{Hippocampal ROI Activations}

Two LMTLR patients had no remaining posterior HIP due to atrophic changes after surgery. In LMTLR patients, activations in the right HIP contralateral to the resection side during word encoding were positively correlated with the postoperative verbal immediate recall scores and postoperative changes in verbal immediate recall scores ( $\mathrm{r}$ $=0.498, \mathrm{p}<0.05 ; \mathrm{r}=0.558, \mathrm{p}<0.05$, respectively; Fig. 3A). In RMTLR patients, activations in the contralateral left HIP during figure encoding were positively correlated with the postoperative changes in the visual immediate and delayed recall scores $(r=0.529, p<0.05 ; r=0.540$, $\mathrm{p}<0.05$, respectively; Fig. 3B). In addition, despite failure to achieve a conventional statistical significance, there was a trend toward a significant positive correlation between the activations in the contralateral left HIP during figure encoding and the postoperative visual immediate and delayed recall scores $(r=0.423, p<0.1 ; r=0.409, p<0.1$, respectively). No significant correlations were identified during the figure encoding in the LMTLR group or during word encoding in the RMTLR group.

As for other clinical variables, the ipsilateral left hippocampal activations in the LMTLR group during word encoding and the ipsilateral right hippocampal activations in the RMTLR group during figure encoding exhibited a negative correlation with age at seizure onset (LMTLR, $\mathrm{r}$ $=-0.520, \mathrm{p}<0.05 ;$ RMTLR, $\mathrm{r}=-0.470, \mathrm{p}<0.05)$ and $\mathrm{a}$ 


\section{A. Word Encoding (Correct > Incorrect)}

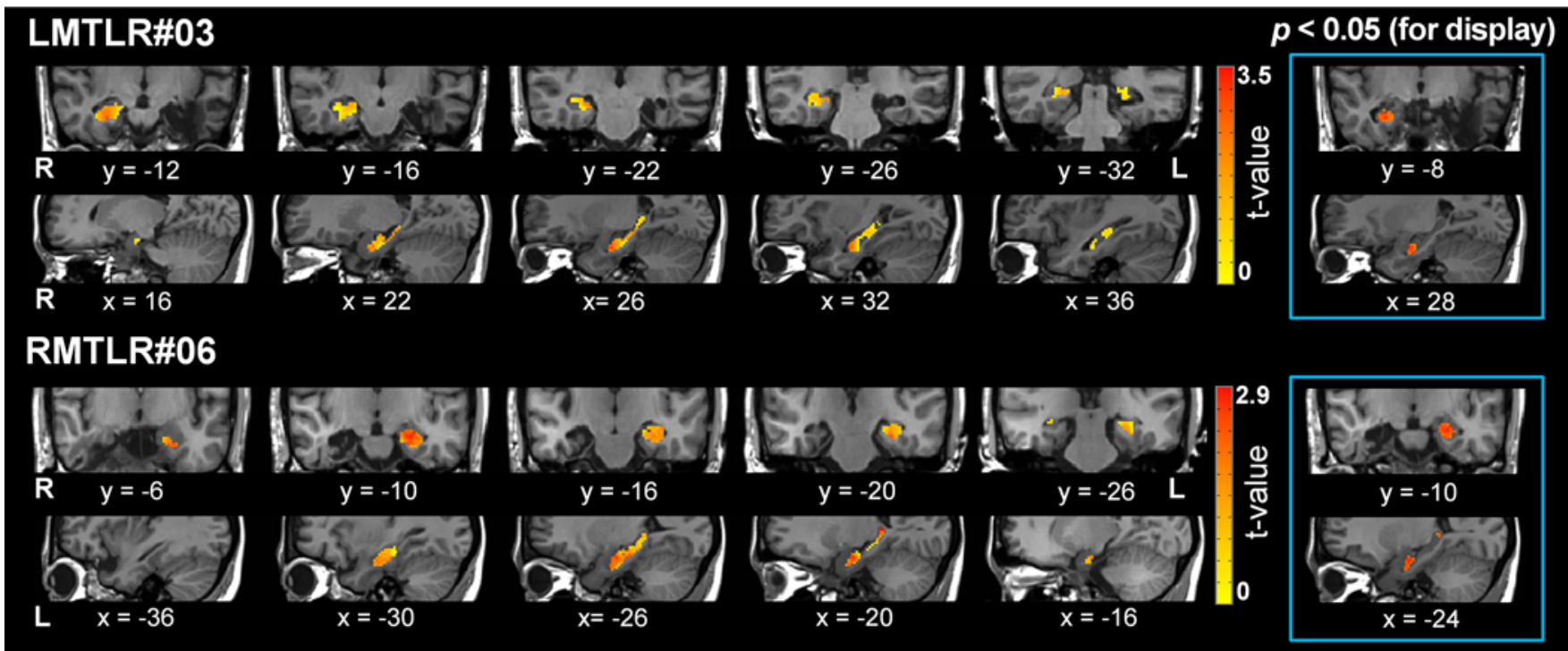

\section{B. Figure Encoding (Correct > Incorrect)}

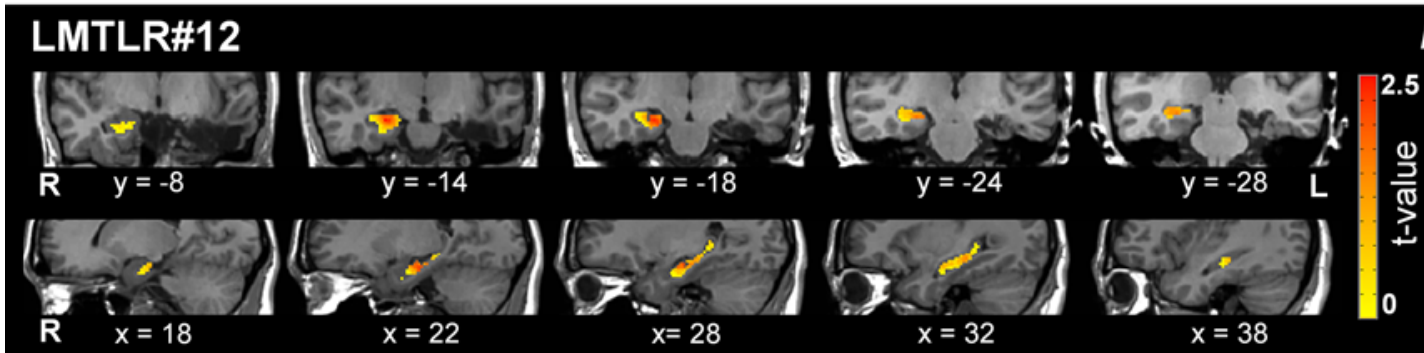

$p<0.05$ (for display)

\section{RMTLR\#18}
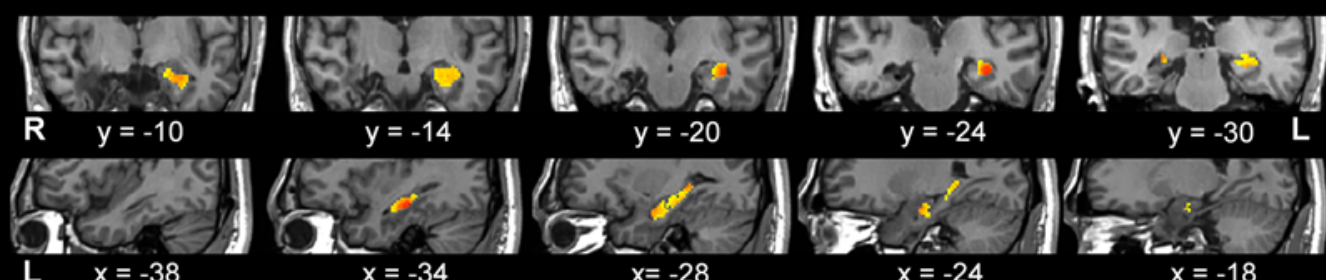

$y=-20$
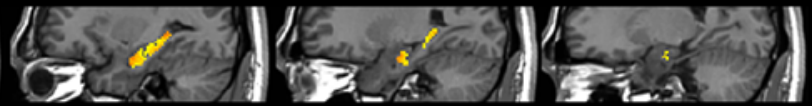

$x=-34$

$x=-28$

$x=-24$

$x=-18$

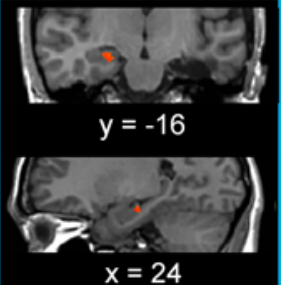

FIG. 2. Examples of activated voxels in the hippocampal ROls during memory encoding (correct > incorrect). A: Hippocampal activation during word encoding in two cases, one where the patient had undergone LMTLR (LMTLR\#03) and the other where the patient had undergone RMTLR (RMTLR\#06). B: Hippocampal activation during figure encoding in two cases, one where the patient had undergone LMTLR (LMTLR\#12) and the other where the patient had undergone RMTLR (RMTLR\#18). Survived voxels at $p<0.05$ (uncorrected) are provided for display purposes only (blue box). Figure is available in color online only.

positive correlation with the duration of illness (LMTLR, $\mathrm{r}=0.561, \mathrm{p}<0.05$; RMTLR, $\mathrm{r}=0.559, \mathrm{p}<0.05)$. That is, activations in the ipsilateral HIP were higher in patients with early seizure onset and a longer duration of illness in both groups of patients. Activations of the ipsilateral remnant HIP had no relationship with any of the memory scores.

\section{Discussion}

The aim of the present study was to characterize the neural basis that supports effective episodic memory in
MTLR patients with an intermediate-term follow-up of more than 1 year after surgery. Postoperative hippocampal activities during successful verbal and visual memory encoding were calculated and compared with standard neuropsychological memory performances, hippocampal volumes, and other clinical variables, such as duration of illness and age at seizure onset.

\section{Neuropsychological Results}

Both the postoperative IQ and MQ scores increased compared to the preoperative baseline in our patient 
TABLE 2. Neuropsychological results

\begin{tabular}{|c|c|c|c|c|}
\hline \multirow[b]{2}{*}{ Test } & \multicolumn{2}{|c|}{$\operatorname{LMTLR}(\mathrm{n}=17)$} & \multicolumn{2}{|c|}{$\operatorname{RMTLR}(n=18)$} \\
\hline & Preop & Postop & Preop & Postop \\
\hline $\mathrm{FSIQ}^{*} \dagger$ & $95.62(14.75)$ & $109.53(13.82)$ & $94.67(16.86)$ & $106.16(11.90)$ \\
\hline $\mathrm{VIQ}{ }^{*} \dagger$ & $92.75(15.37)$ & $105.00(15.98)$ & $94.89(16.39)$ & $104.06(11.40)$ \\
\hline $\mathrm{PIQ}^{*} \ddagger$ & $100.13(13.87)$ & $114.76(10.62)$ & $95.89(15.92)$ & $108.33(12.96)$ \\
\hline$M Q \ddagger \S$ & $89.06(12.66)$ & $98.12(11.61)$ & $90.25(11.30)$ & $105.05(9.83)$ \\
\hline Verbal immediate recall†§ף & $8.71(2.11)$ & $9.76(2.02)$ & $9.61(2.50)$ & $12.33(2.11)$ \\
\hline Verbal delayed recall $\ddagger \S \rrbracket$ & $5.59(3.76)$ & $8.47(4.65)$ & $7.67(3.71)$ & $11.67(2.38)$ \\
\hline Visual immediate recall†§币 & $9.88(2.89)$ & $12.00(1.87)$ & $8.50(2.96)$ & $10.06(1.67)$ \\
\hline Visual delayed recall†§ণ & $9.71(3.02)$ & $11.47(2.70)$ & $8.22(2.84)$ & $9.83(2.31)$ \\
\hline
\end{tabular}

Values are presented as the mean (SD).

* Preoperative versus postoperative, significant differences in LMTLR, $p<0.001$.

$\dagger$ Preoperative versus postoperative, significant differences in RMTLR, $p<0.05$.

$\ddagger$ Preoperative versus postoperative, significant differences in RMTLR, $p<0.001$.

$\S$ Preoperative versus postoperative, significant differences in LMTLR, $p<0.05$.

ๆ LMTLR versus RMTLR postoperative, significant differences, $p<0.05$.

groups. Although stable or declined cognitive function following MTLR has been reported, improvement in IQ and MQ after surgery has also been consistently reported..$^{14,21 \text {, }}$ ${ }^{24,28}$ The first possible reason for this improvement in the present study is the patients' selection criteria, which included patients with an IQ of over 70 at the most recent examination and excluded patients with impaired postoperative memory function. It has been suggested that a higher IQ provides a capacity for the development of an effective compensatory memory after surgery. ${ }^{3,9}$ In the present study, most of the patients (83\%) had a normal range of preoperative IQ (IQ $>90$; average level 23; $80<$ IQ $<89$, low-average level 6). Another possible reason for this improvement could be attributed to functional recovery that occurred due to the absence or reduction of seizures and/ or the cessation or reduction of antiepileptic drugs in our patients (57\% of patients ceased taking antiepileptic drugs) after surgical treatment.

It should be noted that all patients displayed a normal (high-average to low-average) range of memory scores and had a favorable seizure outcome (Engel classes I and II) at the time of fMRI scanning. These characteristics allowed us to investigate the neural basis of memory in the special group of postoperative patients who had intact memory function without the contaminating effect of an underlying disease.

\section{Activations in Hippocampus Contralateral to Resection Side}

Postoperatively the HIP, whether on the ipsilateral or contralateral side of the resection, as it supports effective memory function after surgery, is relatively less understood, especially in the intermediate term after MTLR. In the 1-year follow-up studies of unilateral MTLR patients, researchers have found that postoperative activation of the HIP on the side contralateral to the resection was positively associated with postoperative memory performance ${ }^{7}$ and postoperative memory improvement compared to the preoperative baseline..$^{22}$ In line with previous studies, we also demonstrated that contralateral hippocampal activation was positively associated with postoperative memory capacity and memory improvement after surgery. Specifically, postoperative verbal memory performance after LMTLR was positively correlated with contralateral right hippocampal activation during word encoding. In contrast, the postoperative visual memory performance in RMTLR patients was positively correlated with the contralateral left hippocampal activation during figure encoding.

According to the material-specific memory model, verbal memory is generally more dependent on the left (dominant hemisphere) medial temporal lobe (MTL) structures while visual memory is more dependent on the right MTL structures. ${ }^{26}$ However, in the present study, the contralateral right HIP performed the role of verbal memory function of the left HIP in patients who underwent left HIP resection. In contrast, the contralateral left HIP performed the role of the visual memory function of the right HIP in patients who underwent right HIP resection. Regarding the patients who had better postoperative memory capacity and more postoperative improvement in memory function (verbal memory for LMTLR and visual memory

TABLE 3. Results of hippocampal volume measurement

\begin{tabular}{cccccc}
\hline & \multicolumn{2}{c}{$\operatorname{LMTLR}(\mathrm{n}=17)$} & & \multicolumn{2}{c}{$\operatorname{RMTLR}(\mathrm{n}=18)$} \\
\cline { 2 - 3 } \cline { 5 - 6 } Measurement Time & Left HIP & Right HIP & & Left HIP & Right HIP \\
\hline Preop $\left(\mathrm{mm}^{3}\right)$ & $2024.11(883.46)$ & $3273.57(448.49)$ & & $3132.78(541.39)$ & $2601.14(1092.04)$ \\
\hline Postop $\left(\mathrm{mm}^{3}\right)$ & $460.64(382.52)$ & $3239.90(317.39)$ & & $3183.46(507.31)$ & $547.21(281.52)$ \\
\hline
\end{tabular}

Values presented as the mean (SD). 


\section{A. LMTLR}
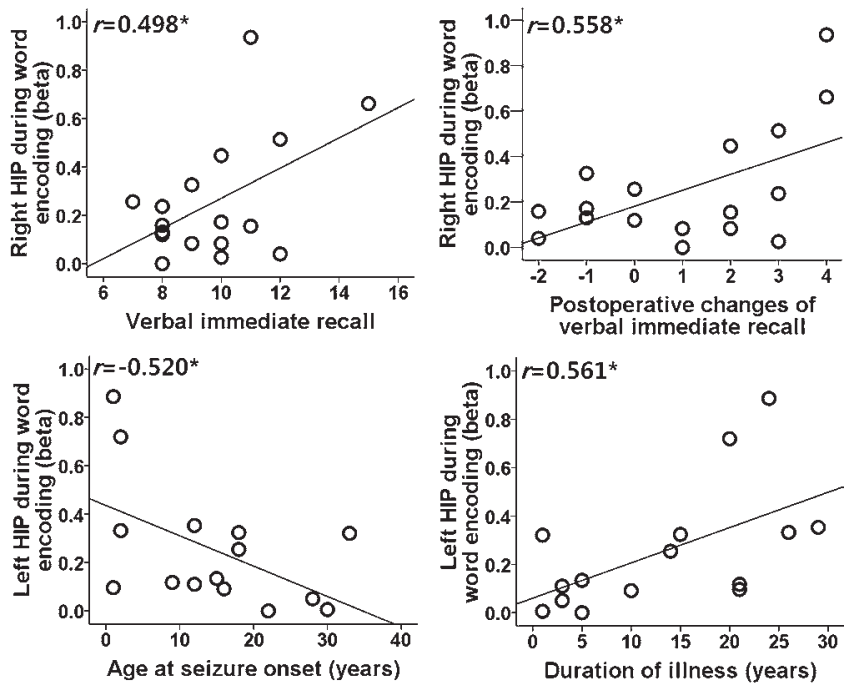

\section{B. RMTLR}
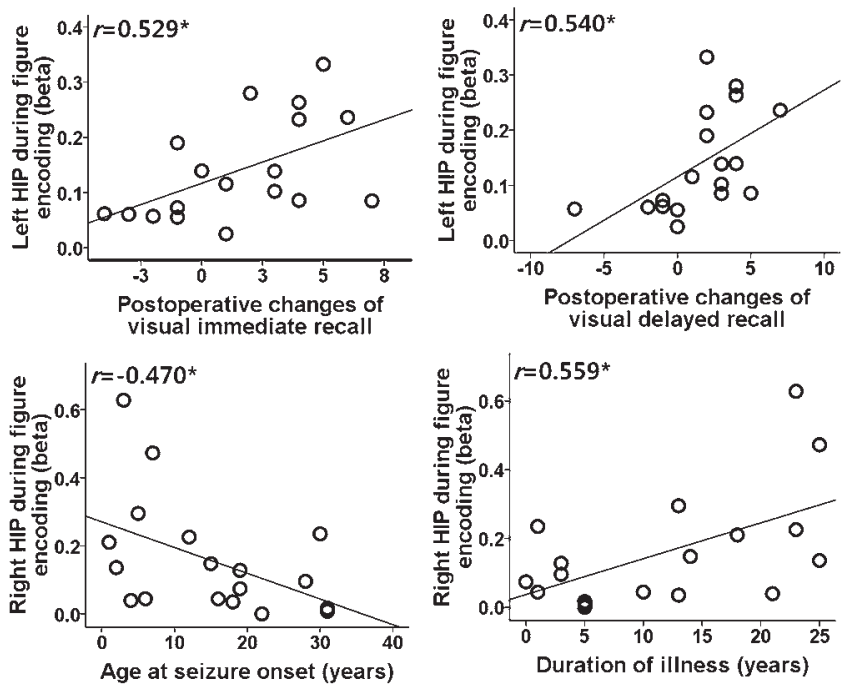

FIG. 3. Relationships of left (A) and right (B) hippocampal activations to clinical variables.

for RMTLR) that displayed stronger activation of the HIP contralateral to the resection, it seems that the materialspecific contralesional HIP may play an important role in effective memory function in patients with a unilateral hippocampal lesion. Moreover, contralateral shift of memory function was not observed for intact-HIP-related memory function, including visual memory for patients who underwent LMTLR and had an intact right HIP and verbal memory for patients who underwent RMTLR and had an intact left HIP.

It is important to note that the mean interval between MTLR and participation in the present study was 6.31 \pm 2.72 years (range $1.25-10.83$ years) but was at most 1 year in the previous studies. In previous reports, neuropsychological follow-up studies consistently indicated that intermediate-term memory outcome following MTLR was stable and even improved, especially in patients who achieved a favorable seizure outcome., ${ }^{1,219,28}$ Regarding the intermediate-term follow-up duration and favorable seizure outcome of our patients, we first showed how the brain supports effective memory function in the intermediate term after surgery, which had previously only been evaluated by neuropsychological tests.

Engagement of the contralateral HIP may play an important role in effective memory function in the intermediate term after surgery for at least those patients with favorable seizure outcome.

\section{Activations in Hippocampus Ipsilateral to Resection Side}

For activation of the ipsilateral remnant HIP, one previous study emphasized its role in postoperative memory function. ${ }^{5}$ At the 4 -month follow-up assessment, changes in the memory scores were related to the relative activationrelated differences in the ipsilateral posterior HIP rather than the contralateral HIP between the pre- and postoperative memory on fMRI. However, since the memory scores were not only compared with the postoperative neural response, it is difficult to characterize the direct relationship between the ipsilateral posterior remnant HIP in the postoperative brain and the individual memory capacity. Moreover, another study by the same research group reported that there is a transient increase of activation in the ipsilateral posterior HIP at 3 months after surgery; however, such an increase is significantly reduced at 12 months after surgery. ${ }^{22}$ Therefore, it is difficult to draw conclusions concerning the role of the ipsilateral remnant HIP in effective memory function after MTLR.

In the present study, greater activation in the ipsilateral remnant HIP was correlated with a longer duration of illness and earlier seizure onset but was without any significant correlation with memory scores or hippocampal volumes. Additional analysis revealed that patients who experienced a seizure at a relatively earlier age had a longer duration of illness (LMTLR, $\mathrm{r}=-0.807$, $\mathrm{p}<0.001$; RMTLR, $\mathrm{r}=-0.777, \mathrm{p}<0.001)$. Although there is no report regarding postoperative activation in relation to the clinical variables, one previous study on preoperative patients with hippocampal sclerosis reported that earlier age at seizure onset was associated with ipsilateral posterior hippocampal activations. ${ }^{23}$ Therefore, we speculate that the effect of the earlier insult in the anterior HIP on ipsilateral posterior hippocampal activation also lasted postoperatively.

Interestingly, using multivoxel pattern analysis, one previous study found that the activity patterns of specific memories were detected in a contralateral HIP, but not a sclerotic HIP, in patients with TLE. ${ }^{26}$ Similarly, the present study demonstrated that activations in the contralateral, but not the ipsilateral, HIP were correlated with the memory performance of MTLR patients. It seems that the ipsilateral posterior HIP is more strongly related to other clinical variables, including the duration of illness and age at seizure onset, rather than individual memory capacity. Taken together, the results of the present study first suggest that the HIP on the contralateral rather than ipsilateral side of the resection supports effective episodic memory function in the intermediate term after surgery.

\section{Limitations and Future Directions}

The present study has several limitations. First, for neu- 
ropsychological testing, simple practice effect from the repeated assessment of IQ and MQ improvement cannot be ruled out. However, the practice effect is known to have minimal or negligible effects on follow-up assessment of at least 1 year postsurgery. ${ }^{25,29}$ The mean retest interval of the neuropsychological examination in our patients was $5.30 \pm 2.73$ years (range $1.0-10.5$ years). Only $5(14 \%)$ of the 35 patients were retested within an interval of less than 2 years. Therefore, the practice effect should be minimal in most of our patients.

Second, due to the retrospective design of the present study, preoperative fMRI studies with the same patients could not be performed. Therefore, we were not able to demonstrate if the effective role of the HIP contralateral to the pathological side on memory function was also observed preoperatively in patients who showed normal memory function postoperatively. An effective shift of memory function to the contralateral HIP might have occurred before and/or after surgery. Preoperative increases of activation in the HIP contralateral to the epileptogenic hemisphere have been regarded as effective reorganization to support preoperative memory function. ${ }^{17}$ Related to postoperative memory performances, one previous study reported that the change in memory performance is related to the pre- and postoperative activation-related differences in the ipsilateral posterior HIP but not in the contralateral HIP. ${ }^{5}$ However, it is also possible that effective contralateral reorganization might have already occurred preoperatively and maintained stable activation after surgery, which in turn resulted in no differences between the pre- and postoperative outcomes in their study.

Additionally, as one previous study demonstrated, the possibility of a postoperative continuous increase of contralateral hippocampal activation cannot be excluded. ${ }^{22}$ Future longitudinal studies investigating hippocampal activity separately for patients with postoperative normal memory and impaired memory function will be required to determine whether effective reorganization to the contralateral HIP might have occurred before or after surgery and whether preoperative activation of the contralateral HIP is a predictive factor for favorable intermediate-term memory outcome, which could potentially help in patient selection and/or counseling.

\section{Conclusions}

In the present study, for the first time, the neural basis of effective intermediate-term episodic memory after resection of unilateral MTL areas was provided. The present study demonstrated that activation in the HIP on the contralateral rather than ipsilateral side of the resection is responsible for effective intermediate-term memory function after surgery for both the LMTLR and RMTLR patient groups. Engagement of the material-specific contralateral HIP, verbal memory for the left-sided surgery group, and visual memory for the right-sided surgery group were observed. Ipsilateral posterior remnant hippocampal activation was related to other clinical factors, such as the duration of illness and age at seizure onset, rather than the postoperative memory capacity.

\section{Acknowledgments}

We thank Soyeon Jun for helping with the experiments. This research was supported by the Basic Science Research Program through the National Research Foundation of Korea, funded by the Ministry of Science \& ICT (2016M3C7A190498, 2018M3C1B8013690).

\section{References}

1. Andersson-Roswall L, Engman E, Samuelsson H, Malmgren $\mathrm{K}$ : Cognitive outcome 10 years after temporal lobe epilepsy surgery: a prospective controlled study. Neurology 74:19771985,2010

2. Baxendale S: Long-term cognitive outcomes after epilepsy surgery in adults, in Malmgren $\mathrm{K}$, Baxendale S, Cross JH (eds): Long-Term Outcomes of Epilepsy Surgery in Adults and Children. Cham: Springer International Publishing, 2015, pp 71-83

3. Baxendale S, Thompson PJ, Duncan JS: Improvements in memory function following anterior temporal lobe resection for epilepsy. Neurology 71:1319-1325, 2008

4. Bell B, Lin JJ, Seidenberg M, Hermann B: The neurobiology of cognitive disorders in temporal lobe epilepsy. Nat Rev Neurol 7:154-164, 2011

5. Bonelli SB, Thompson PJ, Yogarajah M, Powell RH, Samson RS, McEvoy AW, et al: Memory reorganization following anterior temporal lobe resection: a longitudinal functional MRI study. Brain 136:1889-1900, 2013

6. Cardoso MJ, Leung K, Modat M, Keihaninejad S, Cash D, Barnes J, et al: STEPS: Similarity and Truth Estimation for Propagated Segmentations and its application to hippocampal segmentation and brain parcelation. Med Image Anal 17:671-684, 2013

7. Cheung MC, Chan AS, Lam JM, Chan YL: Pre- and postoperative $\mathrm{fMRI}$ and clinical memory performance in temporal lobe epilepsy. J Neurol Neurosurg Psychiatry 80:1099_ 1106, 2009

8. Chung CK, Lee SK, Kim KJ: Surgical outcome of epilepsy caused by cortical dysplasia. Epilepsia 46 (Suppl 1):25-29, 2005

9. Davies KG, Bell BD, Bush AJ, Wyler AR: Prediction of verbal memory loss in individuals after anterior temporal lobectomy. Epilepsia 39:820-828, 1998

10. Engel J Jr, Van Ness PC, Rasmussen TB, Ojemann LM: Outcome with respect to epileptic seizures, in Engel J Jr (ed): Surgical Treatment of the Epilepsies, ed 2. New York: Raven Press, 1993

11. Haag A, Bonelli S: Clinical application of language and memory fMRI in epilepsy. Epileptologie 30:101-108, 2013

12. Helmstaedter C: Neuropsychological aspects of epilepsy surgery. Epilepsy Behav 5 (Suppl 1):S45-S55, 2004

13. Jack CR Jr: MRI-based hippocampal volume measurements in epilepsy. Epilepsia 35 (Suppl 6):S21-S29, 1994

14. Jokeit H, Ebner A: Long term effects of refractory temporal lobe epilepsy on cognitive abilities: a cross sectional study. $\mathbf{J}$ Neurol Neurosurg Psychiatry 67:44-50, 1999

15. Kim H (ed): Rey-Kim Memory Test. Daegu: Neuropsychology Press, 1999

16. Maccotta L, Buckner RL, Gilliam FG, Ojemann JG: Changing frontal contributions to memory before and after medial temporal lobectomy. Cereb Cortex 17:443-456, 2007

17. Richardson MP, Strange BA, Duncan JS, Dolan RJ: Preserved verbal memory function in left medial temporal pathology involves reorganisation of function to right medial temporal lobe. Neuroimage 20 (Suppl 1):S112-S119, 2003

18. Saad ZS, Glen DR, Chen G, Beauchamp MS, Desai R, Cox RW: A new method for improving functional-to-structural MRI alignment using local Pearson correlation. Neuroimage 44:839-848, 2009 
19. Salvato G, Scarpa P, Francione S, Mai R, Tassi L, Scarano E, et al: Declarative long-term memory and the mesial temporal lobe: Insights from a 5-year postsurgery follow-up study on refractory temporal lobe epilepsy. Epilepsy Behav 64 (Pt A):102-109, 2016

20. Schramm J: Temporal lobe epilepsy surgery and the quest for optimal extent of resection: a review. Epilepsia 49:12961307, 2008

21. Shin MS, Lee S, Seol SH, Lim YJ, Park EH, Sergeant JA, et al: Changes in neuropsychological functioning following temporal lobectomy in patients with temporal lobe epilepsy. Neurol Res 31:692-701, 2009

22. Sidhu MK, Stretton J, Winston GP, McEvoy AW, Symms M, Thompson PJ, et al: Memory network plasticity after temporal lobe resection: a longitudinal functional imaging study. Brain 139:415-430, 2016

23. Sidhu MK, Stretton J, Winston GP, Symms M, Thompson PJ, Koepp MJ, et al: Factors affecting reorganisation of memory encoding networks in temporal lobe epilepsy. Epilepsy Res 110:1-9, 2015

24. Wachi M, Tomikawa M, Fukuda M, Kameyama S, Kasahara K, Sasagawa M, et al: Neuropsychological changes after surgical treatment for temporal lobe epilepsy. Epilepsia 42 (Suppl 6):4-8, 2001

25. Wechsler D: WAIS-III: Wechsler Adult Intelligence ScaleThird Edition Administration and Scoring Manual. San Antonio, TX: Psychological Corporation, 1997

26. Willment KC, Golby A: Hemispheric lateralization interrupted: material-specific memory deficits in temporal lobe epilepsy. Front Hum Neurosci 7:546, 2013

27. Winston GP, Cardoso MJ, Williams EJ, Burdett JL, Bartlett PA, Espak M, et al: Automated hippocampal segmentation in patients with epilepsy: available free online. Epilepsia 54:2166-2173, 2013
28. Witt JA, Helmstaedter C: Cognition in epilepsy: current clinical issues of interest. Curr Opin Neurol 30:174-179, 2017

29. Yum T, Park Y, Oh K, Kim J, Lee H (eds): The Manual of Korean-Wechsler Adult Intelligence Scale. Seoul: Korean Guidance Press, 1992

30. Zacà D, Nickerson JP, Deib G, Pillai JJ: Effectiveness of four different clinical fMRI paradigms for preoperative regional determination of language lateralization in patients with brain tumors. Neuroradiology 54:1015-1025, 2012

\section{Disclosures}

The authors report no conflict of interest concerning the materials or methods used in this study or the findings specified in this paper.

\section{Author Contributions}

Conception and design: all authors. Acquisition of data: Jeong. Analysis and interpretation of data: Jeong, Lee, Kim. Drafting the article: Jeong. Critically revising the article: Chung, Lee. Reviewed submitted version of manuscript: all authors. Approved the final version of the manuscript on behalf of all authors: Chung. Statistical analysis: Jeong, Lee. Administrative/technical/ material support: Chung, Kim. Study supervision: Chung, Kim.

\section{Correspondence}

Chun Kee Chung: Seoul National University Hospital, Seoul, South Korea.chungc@snu.ac.kr. 